\title{
Flourishing dogs: the case for an individualized conception of welfare and its implications
}

\author{
Published in Journal of Agricultural and Environmental Ethics 29, 425-438 (2016)
}

\begin{abstract}
Among laypeople, there is a fairly widespread belief according to which there is something wrong with breeding "unnatural" animals, or to breed domestic animals that are too different from their wild ancestors. Influential philosopher Martha Nussbaum argues that animals (including our own species) are entitled to a flourishing life according to the norm for their species. Nussbaum furthermore suggests that in the case of dogs, breed norms as well as species norms are relevant. This suggestion has widespread support among dog enthusiasts as well. I argue that the high diversity of the dog species and the ultimate arbitrariness of breed norms support the thesis that a conception of welfare must be tied to what the individual requires in order to flourish, rather than to collective norms. In the second part of the paper, I discuss the implications that an individualized (but sufficiently sophisticated) welfare conception has for the breeding of dogs for conformation shows, for the pet market and for the performance of various tasks for which we need working dogs.
\end{abstract}

Keywords: Animal welfare; animal breeding; dog breeding; preferentialism; flourishing; species norm; Martha Nussbaum.

\section{Individual conceptions of welfare and their problems}

In this paper, I will refute the thesis that welfare depends on conformation to species or breed norms, and then discuss the implications that an individualized welfare conception has for dog breeding. However, it is easy to see why a collective welfare conception has seemed attractive to some philosophers.

The simplest individualistic conceptions of welfare are revealed preferences preferentialism and a hedonism only concerned with feelings of pleasure or pain (rather than a thicker and more substantial idea of happiness, such as we find in the writings of J. S. Mill). Nussbaum herself presents an alleged counter example against simple hedonism - a German Shepard dog who developed hip problems that made him unable to walk. Nussbaum writes 
that according to the canine species norm, dogs should be able to move around, and thus the species norm supports helping the dog to do just that by giving him a wheelchair. Since he was not in pain, an "individualistic morality", as Nussbaum puts it, would not support helping him - we must appeal to the species norm to show what is wrong with his situation (Nussbaum 2006 p. 365). However, I do not believe that this real life case gives us a good counter example to simple hedonism. No hedonist considers physical pains only. Even if the dog had no physical pains, ${ }^{1}$ his immobility before the wheelchair must have caused him frustration and psychological pain. Thus, we need not appeal to a species norm to explain what was wrong with his situation - even as simple a theory as the version of hedonism discussed here suffices. There is, however, a different problem with hedonism; if we could alleviate his frustration by keeping him drugged, not only on painkillers, but also on a drug that makes him lose interest in everything and be content with his situation, hedonism says that his welfare is high, despite the fact that he is still immobile. This is, of course, an old and familiar problem with simple hedonism - a life constantly doped up on drugs, or, for that matter, a life in Nozick's experience machine, is considered as good a life as one where the individual actually goes out and does things. The idea that an animal has a high level of welfare when she is allowed to satisfy her revealed preferences is equally problematic - an animal who lives in a poor environment might not have been given the opportunity to reveal that many preferences at all, and would need a much richer environment to fully flourish. Lennart Nordenfelt has a theory of animal welfare that somewhat diminishes this problem. He writes that animals have certain preferences that are vital to them, and unless those preferences are satisfied, the animal cannot be happy/have a high level of welfare (Nordenfelt 2006 pp. 147, 151-155). Still, beyond that, happiness is relative to the opportunities with which the animal has been presented. If a certain animal, counterfactually, would become overjoyed from doing activity A, but she has never had the opportunity to A, she might still count as completely happy (ibid. p 170). As Richard Haynes points out in his critique of Nordenfelt, this is still a bit problematic. It seems counter intuitive that one could ensure a high level of welfare for an animal by making certain that the animal never encounters situations or have the chance to try out activities that would fill her with joy (Haynes $2008 \mathrm{pp}$. 120-121).

\footnotetext{
${ }^{1}$ Perhaps he was on good painkillers - otherwise it is hard to believe that his hip problems where severe enough to make him immobile but did not cause him pain.
} 
I believe, however, that these problems can be solved. We might opt for a preference satisfaction theory of welfare according to which preferences must be authentic, along the lines developed by Sumners for humans or Haynes for non-human animals (ibid pp. 143-155). A high level of welfare requires the absence of deception and oppressive socialization, opportunities to develop one's personality, interests and preferences, and then that one's preferences also are satisfied. Alternatively, we might opt for a perfectionist, Aristotleinspired theory, but one focusing on individuals and what perfection would be for this particular animal rather than on species or breed. Such a theory might end up being very similar to Haynes' idea of authentic preference satisfaction - plausibly, an animal has achieved individual perfection and flourishes when she has had the opportunity to develop preferences free from deception and oppression, and then satisfy them.

Now, Nussbaum also argues for the importance of species norms because an individual can only flourish as part of a certain society or way of life. She argues, for instance, that a human with the same intellectual capacities as a chimpanzee has a lower level of welfare due to the problems her intellectual disability will cause her in human society, problems that the chimpanzee in chimp society does not suffer from. However, insofar as she has an authentic preference for taking part in human society an individualized welfare conception can still explain what her problem is, but also allow for the possibility that certain individuals might flourish very well among members of a different species than their own (Kymlicka and Donaldson 2011 pp. 96-98). An individualistic welfare conception can easily incorporate taking part in a certain society within the individual's flourishing or among her authentic preferences, while allowing for the possibility that some individuals might flourish among members of a different species than their own.

I will not delve deeply into possible problems with individual welfare conceptions for animals and possible solutions to these problems, but merely note that I believe these problems to be surmountable, and something along the lines of Haynes' theory to be on the right track. I will rather, in the first part of this paper, argue against welfare conceptualized in relation to a species norm or breed norm. What I will argue is that the case of the dog, this thoroughly domesticated and incredibly diverse species, shows that welfare conceptions that apply to species norms are untenable, and breed norms are ultimately too arbitrary. This is important since reference to a species norm might seem highly plausible when considering less diverse species, and many laypeople and dog enthusiasts simply assume that a species and/or a breed norm is relevant for animal welfare. The latter part of this paper will examine the implications of an individualized welfare conception; specifically, when breeding for 
conformation shows, the pet market and various working dog tasks might compromise the welfare of the dogs bred. Dog enthusiasts who, e.g., lament the effects that the popularity of conformation shows have on dog populations, often fail to distinguish between what actually negatively impact canine welfare and what merely is a problem for them, the human dog enthusiasts. Furthermore, it is often assumed that there are no conflicts between breeding for working capacity and breeding for welfare, but I show that there can be such conflicts.

\section{The irrelevance of being able to survive or reproduce in one's natural environment}

It is not uncommon for people to critique the breeding of domestic animals with extreme bodies by pointing out that they differ much from their wild ancestors and would not be able to survive in nature. There are scholars, too, who argue for something similar (e.g., Bovenkirk et al. 2002 pp. 16-17, Midgley 2000 p. 11) $)^{2}$ and it is thus worthwhile to examine this view first before we move on to Nussbaum's theory of flourishing rather than just surviving. This kind of animal welfare conception might seem plausible, since it seems to imply that animals that, e.g., move with difficulty or have breathing problems, and therefore would not be able to survive in the wild, have a low level of welfare. However, the case of the thoroughly domesticated dog shows that this welfare conception is untenable. It either rules out too little, making it useless, or it rules out too much.

Dogs, as perhaps no other domesticated species, raise the question of what counts as their "natural environment". The dog is a sub-species of the wolf. Does this mean that the dog's natural environment is the environment of the wolf, since they are of the same species, or that the dog's natural environment is in the care of us humans, since it is a separate sub-species?

${ }^{2}$ Since I here refer to authors discussing bio-engineering of animals, it is worth noting that I do not, in this paper, attempt to lay out a full and comprehensive theory of animal ethics. I do not believe that the ability to survive in one's natural environment is intrinsically valuable in any way at all, for reasons that will be clear throughout this subsection. However, for all that I say, we might have duties to animals that are not directly related to their welfare; duties that are rather related to respect and integrity, and we might have general duties to nature as a whole besides our duties to individual animals and/or indirect duties that makes it wrong to do certain things. It might therefore still be morally wrong, for instance, to create non-sentient chickens for meat production that do not have a low level of welfare since the concept of welfare does not apply to them. 
Both suggestions cause problems. It is quite obvious that we do not get anywhere in analysing canine welfare if we suggest that their welfare requires that they are able to survive in our care. Dogs can be quite seriously disabled and suffer from various pains and still live fairly long lives in the care of humans. Dogs might furthermore be unable to mate without assistance, and yet have lots of offspring. Thus, a welfare conception according to which canine welfare requires that they can survive in our care is simply useless; it rules hardly anything out. If we suggest, instead, that a dog does not have a high level of welfare unless she is capable of surviving in the environment of the wolf, we get some really counter intuitive results. Firstly, some dog breeds that are generally healthy and long-lived would still, for various reasons, fare ill if they were forced to sleep outdoors and hunt for their own food. Their coats may be too thin for sleeping outdoors, and they may not be good hunters. According to an ability-to-survive-in-the-wild conception of welfare, these dogs have a low level of welfare despite their good health and long lives. ${ }^{3}$ On reflection, a conception of welfare that hinges on how you would survive under purely counterfactual conditions is counter-intuitive. Suppose that in a hypothetical, post-apocalyptic world, I would not be able to survive unless I was skilled in fixing cars and other pieces of machinery. This might very well mean that my equally unskilled counterpart has a low level of welfare in a possible, postapocalyptic world, but it hardly means that I have a low level of welfare in this world. Likewise, well-fed and happy family dog can have a high level of welfare even if her abandoned counterpart in a different possible world does not.

However, this is probably an area where intuitions differ. Some people are very fond of talking about how wrong it is to breed "unnatural" animals and romanticize the lives of wild animals in nature; those people might find the implication that, say, all pinscher dogs have a low level of welfare because they have thin coats completely plausible. Still, even those people will probably find it hard to swallow the implication that very fearless dogs, such as many German Shepard dogs from bloodlines bred primarily for police and army work, have lower welfare than otherwise similar but more fearful dogs, or that people-friendly dogs have lower welfare than otherwise similar but more shy dogs. But this is implied by any theory of welfare that makes welfare dependent on the ability to survive in the wild; a wild animal

${ }^{3}$ That is, on a pre-theoretical and intuitive understanding of what it means to be healthy; they are not in pain, move around without difficulty, rarely need to be treated by veterinarians and so on. A philosopher who believes that welfare is partly constituted by an ability to survive in the wild might define health in similar terms, and argue that these dogs are unhealthy. 
cannot be too fearless, or she will quickly get in trouble. She must be shy of people, since a wild animal who approaches people, at least when the animal herself is as big and scary as the wolf, will end up being shot and killed. Even if we take people completely out of the equation and focus only on "nature" (I do not really believe that it is plausible to draw a line between nature on the one hand and people on the other, but let us leave this problem aside for the moment), a wolf with no shyness for strangers might get into trouble with other members of her own species. If fearlessness and friendliness in a domesticated animal makes her a better working dog as well as giving her a more happy-go-lucky personality, but does not lead her into trouble in her domesticated life, it seems very counterintuitive that these traits would detract from her welfare, merely because it is bad for a wolf to be too fearless or too friendly with strangers. Thus, the example of dogs shows that being able to survive in one's natural environment cannot be part of an animal's welfare. Let us, therefore, move on to Nussbaum, and her idea that animals must be able to flourish according to their species norm.

\section{Flourishing according to a species norm}

If a high level of welfare for a dog requires that she must flourish according to the species norm for wolves, we get the same problems as discussed above. However, suggesting that she must flourish according to dog norms, norms for the sub-species rather than the species, might solve some problems that we encountered when we considered that she must be able to survive in our care. A dog with a host of illnesses and disabilities might very well be capable of surviving and reproducing in our care, but do not flourish. We might debate the details of what it means to flourish, but for a dog, this plausibly requires that she is naturally (rather than drugged) free from physical pains and psychological suffering, and in addition able to walk, jump and run, live in human society without too much fear, associate with people without too much shyness, interact with other dogs and use all her senses. This is obviously not to say that dogs that fall short on one or more of these points ought to be euthanized; when a dog's life is no longer worth living is a difficult question, but it is clear that a dog's life can be fairly good even if it is not completely flourishing. We ought to, however, strive as far as possible for breeding dogs that fully flourish, and enable dogs already born to do so. Furthermore, although dogs with different interests and hobbies might all flourish, flourishing plausibly requires that they have interests or preferences at all - that they are not apathetic and listless. A variety of illnesses, disabilities and personality traits that experienced and conscientious dog people intuitively consider detrimental to canine welfare will be caught by 
this welfare conception. Still, some problems remain with the "welfare as flourishing according to the species norm" conception.

It is evident that many small dogs can have a high level of welfare according to a canine species norm. Many small dogs are free of physical pains and psychological suffering, and can walk, jump and run, live in human society without too much fear, associate with people without too much shyness, interact with other dogs, use their senses and have interests. Now, imagine a Siberian husky who is born with a certain kind of dwarfism. She never grows past the size of a miniature pinscher. This unexpected mutation does not cause her pain or affects her in any other way; physically, she is quite comparable to an extra fluffy miniature pinscher. Many dog enthusiasts will have the intuition that her small size is a welfare problem. It is not merely the case that she does not look breed typical. A Siberian husky with floppy ears would not look breed typical. We can imagine a sleigh dog enthusiast who uses a husky with floppy ears in his breeding program, arguing that she is such an extraordinary sleigh dog that she needs to pass on her genes despite her ear mutation. If other breed enthusiasts criticize him for using a non-typical dog in a breeding program, we can see him shrugging it off as a "mere beauty fault". The imagined dwarf-sized Siberian husky seems a more serious matter. Here, dog enthusiasts might be prone to say "poor dog". Here, a breeder who used the dwarf in a breeding program could not plausibly shrug the dwarfism off as a "mere beauty fault". The dwarf-sized Siberian husky cannot run with the pack through thick snow or pull a sleigh, and we might argue that this detracts something from her welfare. Even if she is, physically, comparable to a fluffy miniature pinscher, she seems to lack something that the miniature pinscher does not; she is a Siberian husky, and she was meant for something more than merely going on walks and playing with humans or other dogs. While her parents and siblings keep on pulling sleighs with glee, she must be rehomed as an ordinary family dog, and this seems problematic.

Now, an adherent to simple hedonism or revealed preferentialism might not see any problem. The adherent one of those simple individualist welfare conceptions will say that if the dog can be made to feel content living the life of an ordinary family dog, there is no problem here. However, the adherent to a more Aristotle-inspired welfare conception wants a welfare conception that captures our intuitive sense that a life can be, even if not horrible (it is evident that this Siberian husky need not have a horrible life at all, but can have a fairly good one), in some way stunted, less than it ought to be. The husky's life as an ordinary family dog is intuitively stunted in this way (whereas the miniature pinscher's corresponding lifestyle is not). 
Nussbaum is not insensitive to this idea. She writes that it is wrong not to educate and train a border collie (where this is presumably not wrong for all breeds; 2006 p. 397). This suggests that she is open to the idea that for such a diverse species as the dog, there are not only a species norm for welfare, but also breed norms. However, breed norms are ultimately too arbitrary to be part of a welfare conception.

\section{Flourishing according to a breed norm}

At first glance, breed norms might not seem that complicated. Many breeds have a particular task for which they are bred; we can argue that their flourishing requires that they can perform this task. Siberian huskies must have the ability and opportunity to pull sleighs, and Labrador retrievers to go on bird hunts and fetch shot birds. There are, of course, countless Labradors who live as ordinary family dogs and who also have no actual talent for or interest in hunting. Many dog enthusiasts, however, find a welfare conception according to which these family Labradors have a lower welfare than their field type, actively hunting cousins perfectly plausible. They will insist that although ordinary family Labradors need not have bad lives, there is something tragic about them, since they cannot do what they were meant to do.

But what about all show bred cocker spaniels, who do not have what it takes to function as hunting dogs in the traditional spaniel way? Since the show type cocker spaniel has been bred apart from the field type and used as a pure companion dog for so many decades, few dog enthusiasts today seem to regard them as tragic merely because they have lost their hunting abilities. You can still find dog people who hold this view on cocker spaniels, though. But if we look at the Cavalier King Charles spaniel, you would be hard-pressed to find anyone who laments their lack of hunting abilities (their small size, their lack of the right drives and so on), and regard this lack as a welfare problem in itself, quite apart from commonly recognized health issues within the breed. Yet, the Cavalier is descended from hunting spaniels. But since it was hundreds of years since the ancestors of the Cavalier were used for hunting, its ancestral job seems to have become irrelevant for its welfare. However, on reflection, it seems too arbitrary that the mere passage of time would make a difference as to whether a certain quality or lack thereof would affect the welfare of individuals of a certain breed.

Furthermore, we might ask how to regard mixed breeds. Is their welfare solely determined by factors affecting the flourishing of the $\operatorname{dogs} a s \log s$, and not at all impacted by what breeds went into the mix? For instance, if a Border collie and a Labrador retriever has puppies, is it the case that the parental animals' welfare depend to some extent on their herding and hunting abilities respectively, whereas neither ability plays any part in the welfare of the offspring? 
This seems a bit strange and arbitrary, but there is no plausible alternative either. Must the offspring be capable of doing both, in order to have a high level of welfare? Must a mix between Border collie, Labrador retriever, German hunting terrier and Siberian husky be capable of herding, hunting as a gun dog, hunting as a terrier and pulling a sleigh in order to have a high level of welfare? Ultimately, the idea that a dog must have the ability to flourish in her breed typical task is untenable. Still, the intuition that dwarf-size is problematic in a Siberian husky albeit not in a miniature pinscher is an important one. Siberian husky individuals quite often, if not always and necessarily, have other flourishing conditions than miniature pinscher individuals.

\section{Flourishing as an individual}

In order to flourish as an individual, a dog (or any other animal) must have had the opportunity to develop her preferences without deception and oppression, and then satisfy them. In a dog, these preferences plausibly involves, for a dog, the same abilities and activities lined up in the earlier discussion of a canine species norm. One might debate whether using all their senses can be among the preferences of, e.g., a dog who was born deaf. Yet, she plausibly has a preference for smooth interaction with humans and other dogs, that to some extent is frustrated because of her deafness (the exact extent will of course depend on how flexible and accommodating the humans and dogs around her are, but the extent is plausibly never zero). An individualized welfare conception implies that there are at least conceivable if not actual circumstances where deafness does not detract from a dog's welfare at all, but I do not believe this result to be problematic - if she really has no authentic preferences frustrated, her having a high level of welfare is the right result.

Let us now return to the case of the dwarf-sized Siberian husky. Her parents and siblings pull sleighs with glee, whereas she is too small. What is the actual welfare problem here? What is our intuition, according to which she has a welfare problem that a similarly sized miniature pinscher lacks, actually about? I think that dog people, including myself, who feel that the Siberian husky has a lower level of welfare due to her size, feels this at least in part because we do not imagine that she is quite as happy as a dog who has been bred to be a pet and nothing else, like a miniature pinscher, is. She might have a good life. She might satisfy all her revealed preferences. But although she is not be the victim of oppressive humans, but her circumstances are still oppressive. If she, counterfactually, had been normal-sized and allowed to pull a sleigh with a pack of sleigh dogs, she would have experienced a thrill and a happiness that is now out of reach for her. That is her great loss. That is why her small size 
constitutes a welfare problem. But if I am right in this, her situation can be fully accounted for without appealing to a breed norm. The fact that she would be thrilled and supremely happy from pulling a sleigh over vast snowy fields with a pack of other sleigh dogs is a psychological fact about this particular individual. An individualized theory of flourishing or a sophisticated preferentialist one can account for what is wrong with her situation.

Now, many dog people would regard her as equally tragic, perhaps more tragic, if we imagine that she was not only small in size, but also psychologically similar to a miniature pinscher or some other small companion dog. She would be more tragic, they would think, because she is even further from the breed norm for polar dogs. We might imagine that she is descended from many generations of "show bred" huskies who have lost most of the drives necessary for a sleigh dog, and she was, in addition, born with this dwarfism mutation. However, I believe that this intuition is unreliable, and we can see why by considering the case of Cavalier king Charles spaniels and other breeds which were descended from working breeds a very long time ago. If the fact that Cavaliers are small and lack the drives necessary for a good hunting spaniel is not tragic today, why would it have been tragic at some point in the past, when their hunting origin was closer in time? And at which point did it cease to be tragic? Considering the ultimate arbitrariness of which breeds count as working breeds even though their members might be popular as pure companion dogs, and which breeds count as companion breeds, shows us that these intuitions cannot be relied upon.

I conclude that the case of dogs, this thoroughly domesticated and incredibly diverse species, shows that the welfare of an individual does not depend on how closely she conforms to either a species or a breed norm - it depends solely on facts about this individual. An individual has a high level of welfare when she is allowed to develop her personality and interests free from oppression, and then has her preferences satisfied - or, as we might also put it, when she flourishes as an individual. The afore-mentioned suggestion for what it would take to flourish according to a species norm, that is, being free of physical pains and psychological suffering, be able to walk, jump and run, live in human society without too much fear, associate with people without too much shyness, interact with other dogs and use one's senses, and in general have some kind of interests and preferences to satisfy, will plausibly be part of the flourishing conditions for each dog individual even on this individualized conception, but besides these conditions, different individuals (even when they are members of the same breed) might require different abilities, opportunities and activities to fully flourish because their authentic preferences differ. This is not to say that being a member of a certain breed 
cannot give us important clues to what this individual's welfare will require. If an individual dog is a member of the German Shepherd breed, and is descended from bloodlines bred for police and army work, this gives a strong reason to believe that she will require hard work, and furthermore work that requires her using scent to find people and/or things, in order to fully flourish. Still, although breed membership and being descended from certain bloodlines can give us clues, what ultimately determines an individual's flourishing conditions are facts about her.

Now, let us take a look at the implications this welfare conception has for dog breeding.

\section{Breeding for conformation shows and/or the pet market and canine welfare}

Let us use, as our example, the case of the Labrador retriever. The Labrador is considered a gun dog. However, only a minority within the breed is actually bred for hunting purposes. Most Labradors are bred for conformation shows and/or for the pet market. Show/pet bred Labradors differ from their field type cousins in many ways; they tend, for instance, to be calmer in temperament and have much heavier bodies. Fans of the field type and working dog enthusiasts in general tend to lament this, and might sometimes express pity for show/pet type Labradors. They tend to regard these Labradors as stunted, as not being all that they were meant to be. Let us firstly consider the ways in which breeding for conformation shows or the pet market might actually be detrimental to canine welfare, and secondly the ways in which it is not.

\subsection{Ways in which breeding for conformation shows and/or the pet market compromises welfare}

One obvious way in which breeding for conformation shows might be detrimental to canine welfare is the extensive use of a sire with genetic problems. Imagine that a certain male dog becomes Best In Show in some of the biggest dog shows, and gather an impressive list of titles. However, he does carry genes for hip dysplasia, or skin problems, or certain phobias. Because he is such a great winner and has so many impressive titles, he is used extensively in breeding anyway, and many of his offspring inherit these problems. (If this takes place in a country whose national kennel club demands that animals are tested free of, e.g., hip dysplasia before being used in breeding, it is still usually allowed to use an animal in breeding whose close family members all suffer from this problem as long as the individual himself is free of it, and if the problem is common in his family, he is likely to pass it on to his offspring, even if he does not test positive himself.) 
Another, perhaps equally obvious way, in which breeding for conformation shows or the pet market might be harmful, is when either the beauty standards enforced at shows or popular opinion ${ }^{4}$ favours a look which is directly harmful. This is likely not the case with Labradors today, but we might imagine this happening in the future. Imagine that beauty standards evolve according to which a Labrador ought to be exceedingly wide and heavy, until dogs are bred that have trouble moving around.

Finally, the breeding for a calm temperament might have adverse effects if taken too far. I have claimed that individuals cannot flourish unless they have preferences to satisfy, and I will now elaborate a bit on that condition. Imagine that breeders for generations pick dogs that are perceived as easy to handle for breeding. Both show people and people who merely want a family pet might prefer calm dogs, dogs that do not demand too much attention, dogs that do not require much training because they simply lack an interest in hunting cats and other animals, running up to people and putting their muddy paw prints all over them, stealing food and other actions that are normal to dogs but might be seen as undesirable or problematic by humans. Eventually, this might lead to the breeding of dogs that are not interested in anything at all. Since I have conceptualized a high welfare as having one's authentic preferences satisfied, it is more or less trivial that a dog with hardly any preferences at all will have a lower level of welfare than one who has authentic preferences and sees them satisfied.

Furthermore, there is at least a risk that we inadvertently create depressed dogs if we continuously breed for dogs that are as calm and as uninterested in the world around them as possible. We know that it is possible to breed for depression - lab rats have been bred for that purpose, in order to be test subjects for anti depressant drugs. If a trait can be consciously bred for, it is also possible to inadvertently favour the same trait in one's breeding program. Since depressed individuals are characterized by a "calm" behaviour and general lack of initiative, I believe that we should be vary of going too far in the direction of calmness and general "easiness" in dog breeding.

${ }^{4}$ The case of "teacup Chihuahuas" shows that harmful ideas of what is beautiful or cute might evolve independent of conformation shows. Extremely small Chihuahuas are likely to have worse health than those that are of normal Chihuahua size, and conformation show judges likewise prefer their Chihuahuas to be slightly bigger. Still, some people who are not interested in showing their dogs believe that the smaller the better, and this creates a market for extremely small Chihuahua dogs. 


\subsection{Ways in which breeding for conformation shows and/or the pet market does not compromise welfare}

None of the potential problems discussed in the previous subsection are inevitable results of breeding for conformation shows and/or the pet market. We might, for instance, do health tests not just of breeding animals but of their siblings as well, and decide to breed only healthy individuals from healthy families. We might encourage healthy beauty ideals that are not too extreme. We might complement physical health tests with mental tests, ensuring that dogs used in breeding lack problematic phobias, are not too shy, take an interest in their surroundings and retain some playfulness (although dogs bred for shows or to be pets should not be expected to engage in the kind of wild and hyper-energetic play behaviour that some working dogs are fond of engaging in). Suppose that we do all this, and still produce Labrador retrievers that are unfit to be gundogs. Although far from apathetic, they might lack the motivation to work really hard with anything. They might be too independent-minded and difficult to train for this kind of task. They might be too heavy and therefore too slow for hunting purposes. They might be sensitive to, e.g., heat. As long as this sensitivity is not too extreme, i.e., the dog can still take walks in the heat, but cannot work hard, it does not frustrate any interests of a dog who does not care much for hard work anyway.

Working dog enthusiasts might very well consider these dogs tragic, stunted, being less than what they were meant to be. They simply do not live up to the Labrador retriever breed norm. But as I have argued, welfare does not depend on breed norms. If these Labradors fall short of some norm that working dog enthusiasts and hunters have come up with, then this is a problem for the enthusiasts and the hunters, but not for the dogs themselves. The dogs might be well suited for living ordinary family dog lives, and they might prefer nothing else, not because they have been oppressed, but because they simply are not interested in being gun dogs or working really hard with anything. Dog enthusiasts might lament radical changes in a breed as well as the disappearance of one, since they consider dog breeds to be living cultural artefacts, and radically altering them is as problematic and disrespectful as it would be to alter an old and famous painting in a museum. But this, once again, is a matter of human preferences - our preference for keeping certain cultural artefacts around. Dogs do not have preferences, authentic or otherwise, in this area, and so their flourishing is independent of how much their breed has changed over the years. Changes detrimental to their health is of course detrimental to their welfare, but change in itself is irrelevant.

Humans might also find it problematic if there are too few dogs bred for a certain purpose. Suppose that so few Labrador breeders breed talented gun dogs that hunters can no longer 
find good dogs. This is, once again, a problem for the hunters, but not a problem for the dogs. A dog has a lower level of welfare if she has an authentic preference for hunting (or a preference for hunting or engaging in similar enough activities), or would have had one had she had been allowed to fully develop her personality without oppression, but is prevented from hunting. She does not have a lower level of welfare because she simply lacks this preference.

\section{The ways in which breeding for working capacity might compromise canine welfare}

Finally, on an individualized welfare conception, it is possible that breeding for working capacity might compromise the welfare of the dogs as well. This might come as a surprise; breeding for working capacity is often presented as the antidote to all the physical and mental problems that crop up in the show bred bloodlines in many breeds, and it has been argued in the philosophical literature as well that there are no conflicts in this area (Jeppsson 2014). However, it is possible that a male dog that carry various harmful genes is extensively used in breeding because he is such and outstanding working dog as well. Furthermore, according to the individualized welfare conception here favoured, high welfare requires a kind of harmony between body and soul. That harmony might be greater in a thoroughly show bred or pet bred dog, than in one bred with an eye to working capacity.

A calm, slow and heavy dog might have this body-soul harmony, as might an energetic, swift and light one. An energetic but slow and heavy one, however, is more problematic. Imagine a "dual purpose" breeder who, in her quest to produce Labradors that function tolerably well as hunting dogs besides being conformation show winners, manage to produce dogs with heavy bodies, fairly slow, some heat sensitivity, but with an enormous motivation for hard work. Many working dog enthusiasts will consider her dogs better than her colleague's dogs who have similar bodies but are too calm and unmotivated to use on hunts. They will say that albeit her dogs are too slow and heavy, at least they have what it takes mentally, and that is always something. But insofar as these dogs will feel frustrated when they have trouble running through tough terrain, trouble running at all when it is too hot and so on, they are worse off than dogs who simply lack an interest in this kind of work. We might furthermore conclude that if a breeder wants to turn a show/pet bred population around and make it hard-working again, she should start with the physical part and then move on to mentality and motivation. One might argue that the right mentality and motivation are essential to a good working dog, and one might want to start with what is most essential. Still, 
light and swift dogs with a calm temperament and little motivation are better off than slow and heavy dogs who love to work hard.

Finally, a dog does not merely need the physical abilities necessary to fulfil her authentic preferences in order to have a high level of welfare; she must also be given an opportunity to satisfy them. This is the third way in which breeding for working capacity might be detrimental to canine welfare - if we breed more dogs with, e.g., a strong interest in hunting or hunting-like activities, although there are not enough puppy buyers who are ready to give these dogs a home where they can satisfy this interest. For the sake of canine welfare, breeders must adjust their breeding programs after demand. To a large extent, this obviously already happens, but it is still often claimed among enthusiasts that one should pursue one's ideals without regard for what "the market" demands. Now, breeding to meet the demand of the puppy buyer market can obviously be detrimental to canine welfare as well, as when a demand for extremely small Chihuahuas is met by breeders producing such dogs with no regard for their health. What is needed is a distinction between good demand and overall demand in the economic sense. Overall demand consists of everyone who wants a certain kind of dog, whereas the good demand consists of conscientious people who care about dogs and their welfare and want a certain kind of dog. Breeders must not produce more dogs than can be met by good demand. To some extent, good demand is malleable. How many good, conscientious people want a certain type of dog is not forever written in stone, nor does it only depend on factors that breeders are helpless to affect. Presumably not just overall demand but good demand for a certain breed can rise if the breed is promoted in various ways. Still, good demand is not infinitely malleable. If there is bound to be a large good demand for show bred or pet bred Labradors and only a small good demand for hunting bred ones, we should not lament but laud the fact that there are far more show/pet type breeders than field type breeders.

\section{Conclusion}

Very simple individualized conceptions of welfare such as classic hedonism and revealed preferences preferentialism have obvious problems. Some philosophers draw the conclusion that welfare therefore must be relative to a species norm or perhaps a breed norm. However, such collective conceptions of welfare are untenable for dogs. We cannot use the ability to survive in one's natural environment as a measure of welfare, since this will rule out way too little or too much in the case of dogs, depending on whether we conceive of their "natural environment" as in our care or as in the wild where wolves live. The idea that a dog has a 
high level of welfare when she flourishes according to a species norm is more promising, but ultimately a bit too permissive. We cannot solve the problem either by complementing the species norm with a breed norm, since breed norms are too arbitrary. Instead, I propose that we accept something along the lines of Haynes' welfare conception, according to which an individual has a high level of welfare when she gets to satisfy her authentic preferences. This, however, has certain implications for the breeding of show dogs, pets and working dogs. It allows us to clearly distinguish between what is a problem for human dog enthusiasts and what is a problem for the dogs - these problems do not always coincide. It is a problem for the dogs if they are ill, disabled, suffer mentally from phobias and the like, and, less obviously, if there is a lack of harmony between body and soul, so that they have authentic preferences for activities that they have a hard time doing due to a mismatched body. It is furthermore a problem for the dogs if they have the ability to engage in an activity for which they have an authentic preference, but cannot do so because they lack the opportunity. However, the mere fact that dogs change, or that some dogs are unfit to perform tasks that their ancestors once excelled in, is a problem for dog enthusiasts only, but not for the dogs themselves.

\section{References}

Bovenkirk, Bernice, Frans W. A. Brom and Babs J. Van Den Bergh. (2002) "Brave New Birds: The use of "animal integrity” in Animal Ethics." Hastings Center Report 32 (1),16-22. Donaldson, S. and Kymlicka, W. (2011) Zoopolis. A Political Theory of Animal Rights. New York: Oxford University Press.

Haynes, R. P. (2008) Animal Welfare. Competing Conceptions and Their Ethical Implications. New York: Springer.

Jeppsson, S. 2014. "Purebred dogs and canine wellbeing". Journal of Agricultural and Environmental Ethics. 27 (3),417-430

Midgley, Mary. 2000. "Biotechnology and Monstrosity: Why we should pay attention to the 'yuk' factor”, Hastings Center Report 30 (5), 7-15

Nussbaum, Martha C. 2006. Frontiers of Justice: Disability, Nationality, Species Membership. Cambridge, MA: Harvard University Press, Belknap Press. 